\title{
Anomalous helical edge states in a non-Hermitian Chern insulator
}

\author{
Kohei Kawabata, ${ }^{1,}$, Ken Shiozaki, ${ }^{2}$ and Masahito Ueda ${ }^{1,3}$ \\ ${ }^{1}$ Department of Physics, University of Tokyo, 7-3-1 Hongo, Bunkyo-ku, Tokyo 113-0033, Japan \\ ${ }^{2}$ Condensed Matter Theory Laboratory, RIKEN, Wako, Saitama 351-0198, Japan \\ ${ }^{3}$ RIKEN Center for Emergent Matter Science (CEMS), Wako, Saitama 351-0198, Japan
}

(Dated: October 31, 2018)

\begin{abstract}
A non-Hermitian extension of a Chern insulator and its bulk-boundary correspondence are investigated. It is shown that in addition to the robust chiral edge states that reflect the nontrivial topology of the bulk (nonzero Chern number), anomalous helical edge states localized only at one edge can appear, which are unique to the non-Hermitian Chern insulator.
\end{abstract}

\section{INTRODUCTION}

Over the past decade, remarkable progress has been achieved in phases of matter characterized by the topology of their wave functions [1 15 . Such topological phases were explored in solid-state systems including insulators [6-13, superconductors [14-18, and semimetals 1921], as well as in photonic [22 26] and atomic 27+33. systems, all of which are classified according to spatial dimension and symmetry 34 41. A hallmark of these topological phases is the bulk-boundary correspondence: the topologically protected gapless boundary states appear as a consequence of the nontrivial topology of the gapped bulk. Examples include chiral edge states in Chern insulators [7, helical edge states in quantum spin Hall insulators 8 11, and Majorana zero modes in topological superconducting wires [15].

Recently, there has been growing interest in nonHermitian topological phases of matter both in theory [42 72] and experiment [73 80]. In general, nonHermiticity arises from the exchange of energy and/or particles with the environment [81 84, and several phenomena unique to the nonconservative systems have been theoretically proposed $85-113$ and experimentally observed [114 126]. A key feature of non-Hermitian systems is the presence of a level degeneracy called an exceptional point [127 129], at which eigenstates coalesce to render the Hamiltonian nondiagonalizable. The exceptional point brings about novel functionalities with no Hermitian counterparts such as unidirectional invisibility [95, 116 118] and enhanced sensitivity 98, 102, 125, 126. Recent studies have also revealed that nonHermiticity alters the nature of the bulk-boundary correspondence in topological systems [43, 46, 47, 49, 51, 59, 60, 67. Non-Hermiticity was shown to amplify the topologically protected edge states [46, which were experimentally observed in one dimension $[75,78$, and two dimensions 79]. Furthermore, the presence of exceptional points makes edge states anomalous, so that they are localized only at one edge in a non-Hermitian extension of the Su-Schrieffer-Heeger model (i.e., a non-Hermitian system in one dimension that respects sublattice symmetry) [49. Despite these recent studies, the bulk-boundary correspondence in non-Hermitian systems has yet to be fully understood, especially in two dimensions.

In this work, we investigate a non-Hermitian extension of a Chern insulator and its bulk-boundary correspondence. In Sec. II], we give the topological invariants for a general non-Hermitian system in two dimensions without symmetry (2D class A). In Sec. III, we consider a typical non-Hermitian Chern insulator and provide its phase diagram under the periodic boundary condition. It consists of the gapped phases characterized by the Chern number and the gapless phases characterized by the topological charges accompanied by exceptional points. In Sec. IV] we investigate the topologically protected edge states. The chiral edge states are shown to be robust even in the non-Hermitian systems; they can also be amplified (lasing) due to the presence of non-Hermiticity. Moreover, we find the emergence of the helical edge states localized only at one edge in some phases. These anomalous helical edge states do not have Hermitian counterparts and hence they are unique to the non-Hermitian Chern insulator. In Sec. V] we conclude the paper with an outlook.

\section{TOPOLOGICAL INVARIANTS}

We first provide the topological invariants of a general non-Hermitian system in two dimensions without symmetry (2D class A). If the Hamiltonian $H(\boldsymbol{k})$ is diagonalizable, it can be expressed as

$$
H(\boldsymbol{k})=\sum_{n} E_{n}(\boldsymbol{k})\left|\varphi_{n}(\boldsymbol{k})\right\rangle\left\langle\chi_{n}(\boldsymbol{k})\right| .
$$

Here $E_{n}(\boldsymbol{k})$ is a complex eigenenergy and $\left|\varphi_{n}(\boldsymbol{k})\right\rangle$ $\left(\left|\chi_{n}(\boldsymbol{k})\right\rangle\right)$ is the corresponding right (left) eigenstate [130, 131, which satisfy

$$
\begin{aligned}
H(\boldsymbol{k})\left|\varphi_{n}(\boldsymbol{k})\right\rangle & =E_{n}(\boldsymbol{k})\left|\varphi_{n}(\boldsymbol{k})\right\rangle, \\
H^{\dagger}(\boldsymbol{k})\left|\chi_{n}(\boldsymbol{k})\right\rangle & =E_{n}^{*}(\boldsymbol{k})\left|\chi_{n}(\boldsymbol{k})\right\rangle,
\end{aligned}
$$

and

$$
\begin{aligned}
\left\langle\chi_{m}(\boldsymbol{k}) \mid \varphi_{n}(\boldsymbol{k})\right\rangle & =\delta_{m n}, \\
\sum_{n}\left|\varphi_{n}(\boldsymbol{k})\right\rangle\left\langle\chi_{n}(\boldsymbol{k})\right| & =I,
\end{aligned}
$$

\footnotetext{
* kawabata@cat.phys.s.u-tokyo.ac.jp
} 
with the identity matrix $I$. Here $\left|\varphi_{n}(\boldsymbol{k})\right\rangle$ and $\left|\chi_{n}(\boldsymbol{k})\right\rangle$ are equal to each other in the presence of Hermiticity, but they are not for general non-Hermitian Hamiltonians. If the complex band $n$ is separated from the others (i.e., $\left.\forall \boldsymbol{k}, m \quad E_{m}(\boldsymbol{k}) \neq E_{n}(\boldsymbol{k})\right)$, we can define the Berry connection $\mathcal{A}_{n}^{i}(\boldsymbol{k})$ and the Berry curvature $\mathcal{F}_{n}(\boldsymbol{k})$ of the complex band $n$ as

$$
\begin{aligned}
\mathcal{A}_{n}^{i}(\boldsymbol{k}) & :=\mathrm{i}\left\langle\chi_{n}(\boldsymbol{k}) \mid \partial_{k_{i}} \varphi_{n}(\boldsymbol{k})\right\rangle, \\
\mathcal{F}_{n}(\boldsymbol{k}) & :=\partial_{k_{x}} \mathcal{A}_{n}^{y}(\boldsymbol{k})-\partial_{k_{y}} \mathcal{A}_{n}^{x}(\boldsymbol{k}),
\end{aligned}
$$

and the (first) Chern number as [45, 62.

$$
C_{n}:=\int_{\mathrm{BZ}} \frac{d^{2} \boldsymbol{k}}{2 \pi} \mathcal{F}_{n} \in \mathbb{Z},
$$

where the integration is taken over the first Brillouin zone (BZ). The Chern number remains unchanged as long as the complex band is separated and the Hamiltonian is diagonalizable. Due to the difference between the left and right eigenstates, there is some arbitrariness concerning the definition of the Berry connection. For instance, it can also be defined only by the right eigenstates as $\tilde{\mathcal{A}}_{n}^{i}(\boldsymbol{k}):=\mathrm{i}\left\langle\varphi_{n}(\boldsymbol{k}) \mid \partial_{k_{i}} \varphi_{n}(\boldsymbol{k})\right\rangle$. However, the Chern number does not depend on such choices of the eigenstates and can be uniquely defined 62 .

On the other hand, the Chern number is not welldefined if the Hamiltonian is nondiagonalizable at a wavenumber $\boldsymbol{k}=\boldsymbol{k}_{\mathrm{EP}}$ (i.e., some eigenstates coalesce and linearly depend on each other) 127 129. Instead, in the presence of such a defective point (exceptional point), we can define another topological invariant given by $51,52,62,76,121,122$

$$
\nu\left(\boldsymbol{k}_{\mathrm{EP}}\right):=\oint_{\Gamma\left(\boldsymbol{k}_{\mathrm{EP}}\right)} \frac{d \boldsymbol{k}}{2 \pi \mathrm{i}} \cdot \nabla_{\boldsymbol{k}} \log \left(E_{m}-E_{n}\right),
$$

where the two bands $E_{m}(\boldsymbol{k})$ and $E_{n}(\boldsymbol{k})$ coalesce at $\boldsymbol{k}=\boldsymbol{k}_{\mathrm{EP}}$, and $\Gamma\left(\boldsymbol{k}_{\mathrm{EP}}\right)$ is a closed loop in momentum space that encircles $\boldsymbol{k}_{\mathrm{EP}}$. Here fractional $\nu\left(\boldsymbol{k}_{\mathrm{EP}}\right)$ implies that $E_{m}(\boldsymbol{k})-E_{n}(\boldsymbol{k})$ is multi-valued and that $\boldsymbol{k}_{\mathrm{EP}}$ becomes a branch point in momentum space. The charge $\nu\left(\boldsymbol{k}_{\mathrm{EP}}\right)$ defined as Eq. (7) is topological in that it cannot be changed unless the exceptional point is annihilated.

\section{PHASES AND COMPLEX SPECTRA}

In the following, we consider the non-Hermitian Chern insulator given by

$$
\begin{aligned}
H(\boldsymbol{k})=(m+ & \left.t \cos k_{x}+t \cos k_{y}\right) \sigma_{x} \\
& +\left(\mathrm{i} \gamma+t \sin k_{x}\right) \sigma_{y}+\left(t \sin k_{y}\right) \sigma_{z},
\end{aligned}
$$

where $t, m$, and $\gamma$ are real parameters and we assume $t>0$. In the case of $\gamma=0$, the model reduces to the wellknown Hermitian Chern insulator that is characterized by the Chern number [1 5]. The eigenstates form two

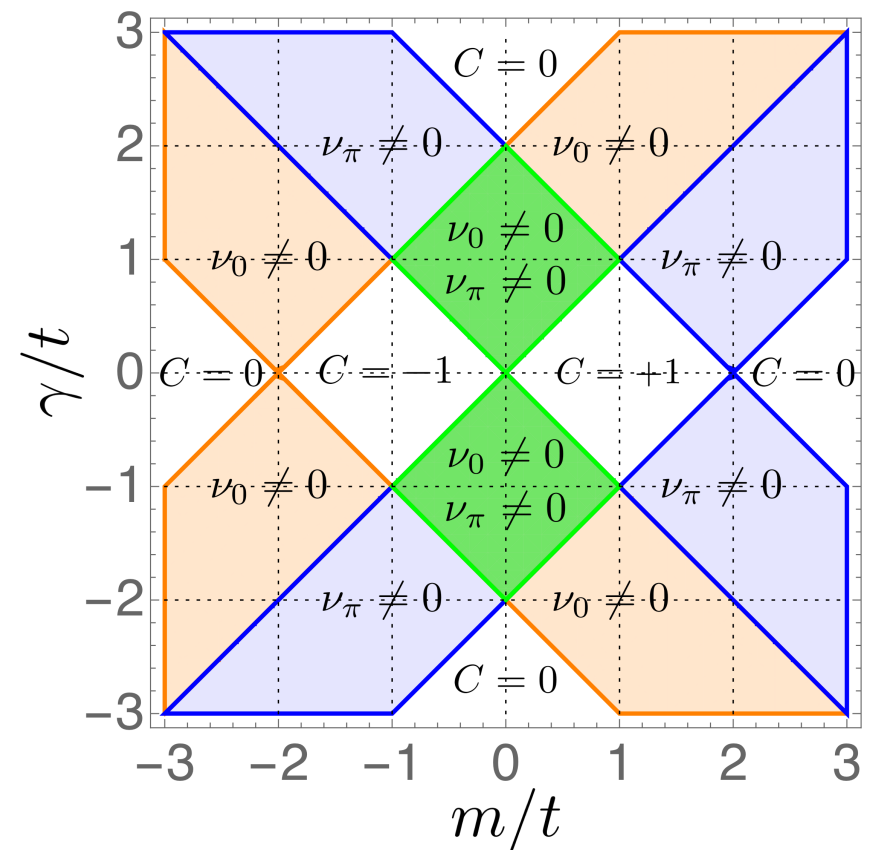

FIG. 1. Phase diagram of the non-Hermitian Chern insulator with periodic boundaries given by Eq. (8). The white regions represent the gapped phases that are characterized by the Chern number $C$. The orange $\left(\nu_{0} \neq 0\right)$, blue $\left(\nu_{\pi} \neq 0\right)$, and green $\left(\nu_{0} \neq 0, \nu_{\pi} \neq 0\right)$ regions represent the gapless phases where pairs of exceptional points appear on $k_{x}=0, k_{x}= \pm \pi$, and both $k_{x}=0$ and $k_{x}= \pm \pi$, respectively.

bands and their complex energy dispersions are obtained as

$$
\begin{aligned}
E_{ \pm}(\boldsymbol{k})= \pm[ & \left(m+t \cos k_{x}+t \cos k_{y}\right)^{2} \\
& \left.+\left(\mathrm{i} \gamma+t \sin k_{x}\right)^{2}+\left(t \sin k_{y}\right)^{2}\right]^{1 / 2}
\end{aligned}
$$

The Hamiltonian becomes defective if and only if

$$
\exists \boldsymbol{k}_{\mathrm{EP}} \in[-\pi, \pi]^{2} \quad \text { s.t. } E_{ \pm}\left(\boldsymbol{k}_{\mathrm{EP}}\right)=0 .
$$

This requires $\sin \left(k_{\mathrm{EP}}\right)_{x}=0$ and reduces to the following conditions:

(1) Exceptional points appear on $\left(k_{\mathrm{EP}}\right)_{x}=0$; Eq. 10 reduces to

$$
\gamma^{2}=(m+t)^{2}+t^{2}+2 t(m+t) \cos \left(k_{\mathrm{EP}}\right)_{y},
$$

where $\left(k_{\mathrm{EP}}\right)_{y} \in[-\pi, \pi]$ exists if and only if

$$
\begin{cases}|m| \leq|\gamma| \leq|m+2 t| & \text { for } m \geq-t \\ |m+2 t| \leq|\gamma| \leq|m| & \text { for } \quad m \leq-t\end{cases}
$$

(2) Exceptional points appear on $\left(k_{\mathrm{EP}}\right)_{x}= \pm \pi$; Eq. (10) reduces to

$$
\gamma^{2}=(m-t)^{2}+t^{2}+2 t(m-t) \cos \left(k_{\mathrm{EP}}\right)_{y},
$$



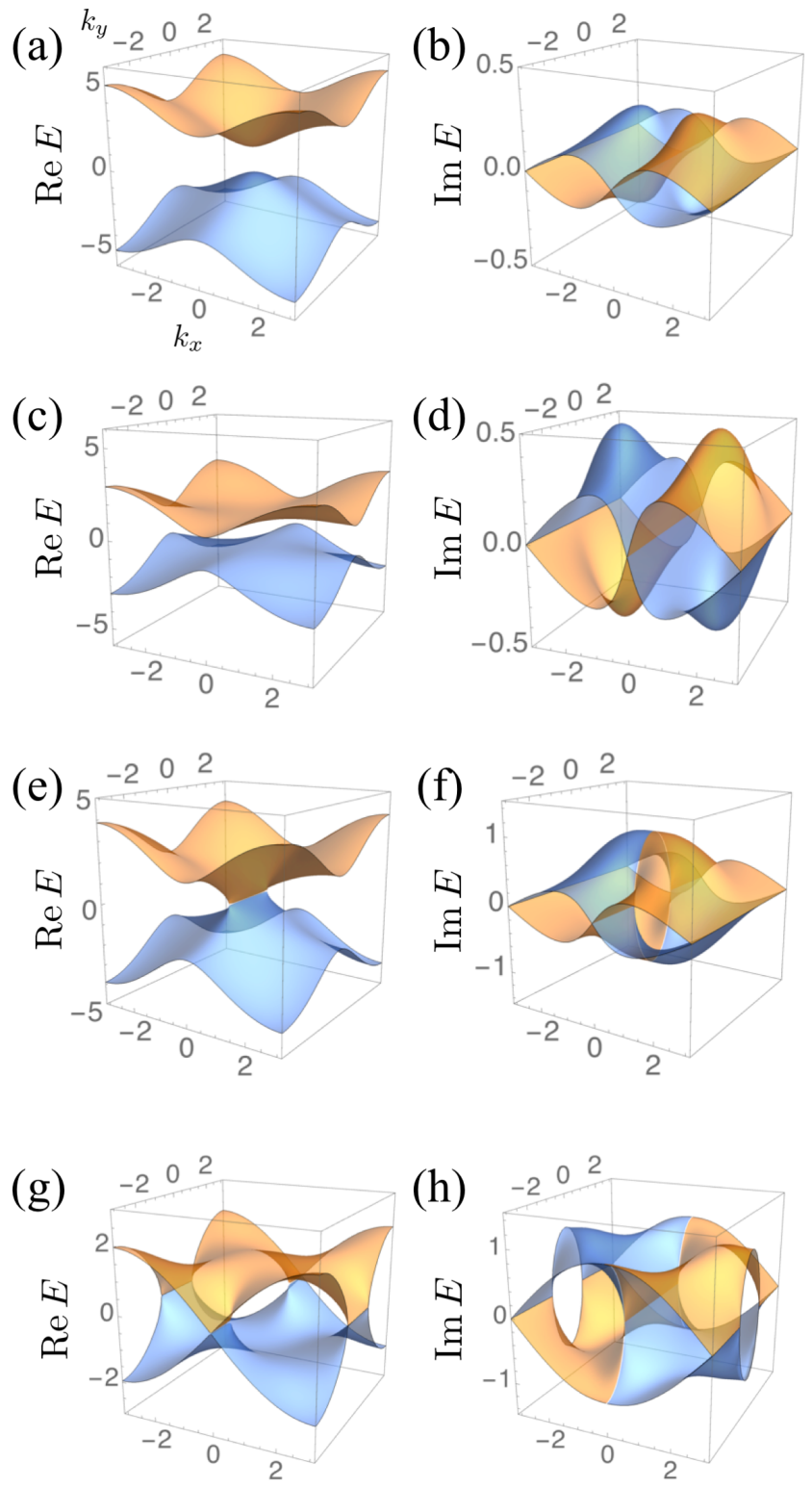

FIG. 2. Complex-band structures of the non-Hermitian Chern insulator $E_{ \pm}=E_{ \pm}\left(k_{x}, k_{y}\right)$ given by Eq. (9). The orange (blue) band represents $E_{+}\left(E_{-}\right)$. (a-b) Real and imaginary parts of the gapped and topologically trivial bands with zero Chern number $C=0(t=1.0, m=-3.0, \gamma=0.5)$. (cd) Real and imaginary parts of the gapped and topologically nontrivial bands with nonzero Chern number $C=-1(t=$ $1.0, m=-1.0, \gamma=0.5$ ). (e-f) Real and imaginary parts of the gapless bands with a pair of exceptional points on $k_{x}=0$ $(t=1.0, m=-2.0, \gamma=1.0)$. (g-h) Real and imaginary parts of the gapless bands with two pairs of exceptional points on both $k_{x}=0$ and $k_{x}= \pm \pi(t=1.0, m=-0.2, \gamma=1.0)$.

where $\left(k_{\mathrm{EP}}\right)_{y} \in[-\pi, \pi]$ exists if and only if

$$
\begin{cases}|m-2 t| \leq|\gamma| \leq|m| & \text { for } m \geq t \\ |m| \leq|\gamma| \leq|m-2 t| & \text { for } m \leq t\end{cases}
$$

Hence we obtain the phase diagram (Fig. 1) and the corresponding complex band structures (Fig. 2). The gapped phases with $E_{+}(\boldsymbol{k}) \neq E_{-}(\boldsymbol{k})$ for all $\boldsymbol{k}$ (Fig. 2 (ad)) are characterized by the Chern number defined by Eq. (6). Similar to the Hermitian counterparts, the Chern number determines the presence or absence of the chiral edge states, as discussed in the next section. Remarkably, the gapped phases are separated by the broad gapless phases that accompany pairs of exceptional points (Fig. 2(e-h)), whereas they are separated by a point in the Hermitian counterpart $(\gamma=0)$. The appearance of such large gapless phases arises from the fact that a level degeneracy requires fine-tuning two parameters for a general $2 \times 2$ non-Hermitian matrix, although it requires fine-tuning three parameters for a general $2 \times 2$ Hermitian matrix 62, 65, 76, 127]; non-Hermitian systems in two dimensions can be gapless without fine-tuning parameters or the presence of certain symmetry. As explained in the last section, the topological invariants can be defined for each exceptional point as Eq. (7). For instance, if there appears a pair of exceptional points at $\boldsymbol{k}_{\mathrm{EP}}=(0, \pm \kappa)$ $(\kappa>0)$ as in Fig. 2(e-f), the energy dispersions near the exceptional points are given by

$$
E_{+}(\boldsymbol{k}) \simeq \sqrt{2 \mathrm{i} t \gamma k_{x} \pm t^{2}(\sin 2 \kappa)\left(k_{y} \mp \kappa\right)} .
$$

Here the square-root singularity implies that the exceptional points $\boldsymbol{k}=\boldsymbol{k}_{\mathrm{EP}}$ become branch points and that the topological charges defined by Eq. (7) are $\nu= \pm 1 / 2$ for $\boldsymbol{k}_{\mathrm{EP}}=(0, \pm \kappa)$. Although the Chern number cannot be defined, we find the emergence of the anomalous helical edge states in these gapless phases, as discussed in the next section.

\section{EDGE STATES}

The hallmark of the Hermitian Chern insulator is the emergence of the chiral edge states that correspond to the nontrivial topology of the bulk [1 $[5$. It is shown that the bulk-edge correspondence in the presence of nonHermiticity is sensitive to the boundary conditions [132]. In the following, we investigate the system with open boundaries only in the $y$ direction, the system with open boundaries only in the $x$ direction, and the system with open boundaries in both $x$ and $y$ directions.

\section{A. Open boundaries in the $y$ direction}

We first consider the following non-Hermitian Chern insulator with periodic boundaries in the $x$ direction and open boundaries in the $y$ direction:

$$
\begin{aligned}
& \hat{H}=\sum_{k_{x}} \sum_{y}\left\{\left[\hat{c}_{k_{x}, y+1}^{\dagger} \frac{t\left(\sigma_{x}+\mathrm{i} \sigma_{z}\right)}{2} \hat{c}_{k_{x}, y}+\text { H.c. }\right]\right. \\
& \left.+\hat{c}_{k_{x}, y}^{\dagger}\left[\left(m+t \cos k_{x}\right) \sigma_{x}+\left(\mathrm{i} \gamma+t \sin k_{x}\right) \sigma_{y}\right] \hat{c}_{k_{x}, y}\right\},
\end{aligned}
$$


(a)

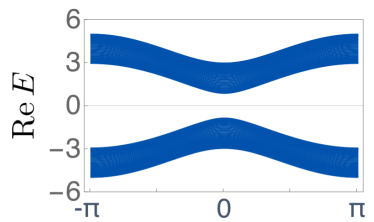

(c)

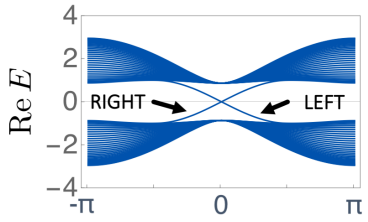

(e)

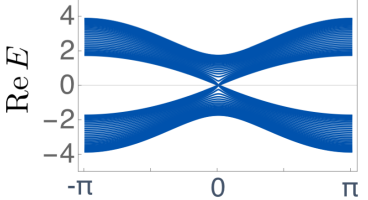

$(\mathrm{g})$

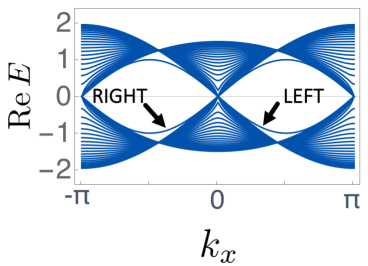

(b)

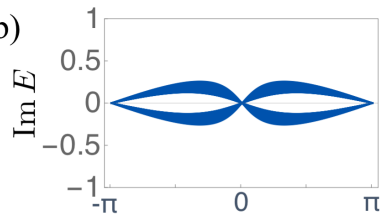

(d)

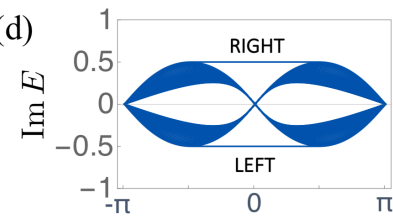

(f)

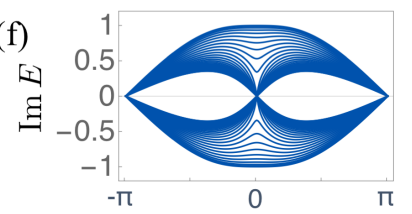

(h)

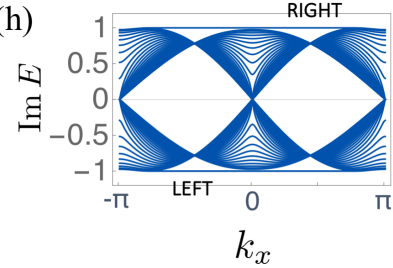

FIG. 3. Complex spectrum of the non-Hermitian Chern insulator given by Eq. (16), which has periodic boundaries in the $x$ direction and open boundaries in the $y$ direction $\left(L_{y}=30\right)$. (a-b) Real and imaginary parts of the complex spectrum in the gapped and topologically trivial phase $(t=1.0, m=-3.0, \gamma=0.5)$. No gapless states appear between the gapped complex bands. (c-d) Real and imaginary parts of the complex spectrum in the gapped and topologically nontrivial phase $(t=1.0, m=-1.0, \gamma=0.5)$. A pair of chiral edge states appears at both edges between the gapped complex bands whose dispersions are $E_{\text {edge }}\left(k_{x}\right)=$ $\pm\left(t \sin k_{x}+\mathrm{i} \gamma\right)$. (e-f) Real and imaginary parts of the complex spectrum in the gapless phase with an exceptional point at $k_{x}=0(t=1.0, m=-2.0, \gamma=1.0)$. (g-h) Real and imaginary parts of the complex spectrum in the gapless phase with exceptional points at both $k_{x}=0$ and $k_{x}= \pm \pi$ $(t=1.0, m=-0.2, \gamma=1.0)$. A pair of chiral edge states appears at both edges, despite the absence of the energy gap.

where $\hat{c}_{k_{x}, y}\left(\hat{c}_{k_{x}, y}^{\dagger}\right)$ annihilates (creates) a fermion with two internal degrees of freedom on site $y$ and with momentum $k_{x}$.

In the gapped phase with the topologically trivial bulk (Fig. 2(a-b)), no gapless states appear between the gapped complex bands (Fig. 3(a-b)). In the gapped phase with the topologically nontrivial bulk (Fig. 2) (cd)), on the other hand, chiral edge states appear, one localized at the right edge and the other localized at the left edge (Fig. 3 (c-d) and Fig. (4). The energy dispersions of these chiral edge states are analytically obtained as

$$
E_{\text {left }}\left(k_{x}\right)=-t \sin k_{x}-\mathrm{i} \gamma, \quad E_{\text {right }}\left(k_{x}\right)=t \sin k_{x}+\mathrm{i} \gamma,
$$

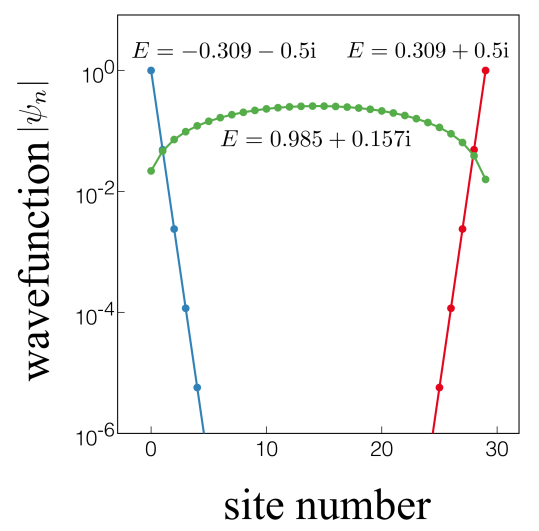

FIG. 4. Wavefunctions of the non-Hermitian Chern insulator given by Eq. (16), which has periodic boundaries in the $x$ direction and open boundaries in the $y$ direction $\left(L_{y}=30\right)$. The system is in the gapped and topologically nontrivial phase that corresponds to Fig. 3 (c-d) $(t=1.0, m=-1.0, \gamma=$ $\left.0.5 ; k_{x}=0.1 \pi\right)$. Whereas the bulk state with $E=0.985+$ $0.157 \mathrm{i}$ (green dots) is delocalized throughout the bulk, the chiral edge states with $E= \pm(0.309+0.5 \mathrm{i})$ (red and blue dots) are localized at both edges.

and the corresponding edge states are given by

$$
\begin{gathered}
\hat{\Psi}_{\text {left }}\left(k_{x}\right) \propto \sum_{y=1}^{L_{y}}\left(-\cos k_{x}-\frac{m}{t}\right)^{y-1} \hat{c}_{k_{x}, y}\left(\begin{array}{c}
1 \\
-\mathrm{i}
\end{array}\right), \\
\hat{\Psi}_{\text {right }}\left(k_{x}\right) \propto \sum_{y=1}^{L_{y}}\left(-\cos k_{x}-\frac{m}{t}\right)^{y-1} \hat{c}_{k_{x}, y}\left(\begin{array}{c}
1 \\
\mathrm{i}
\end{array}\right),
\end{gathered}
$$

which satisfy $\left[\hat{H}, \hat{\Psi}_{\text {left/right }}\right]=E_{\text {left/right }} \hat{\Psi}_{\text {left/right }}$ in the thermodynamic limit $L_{y} \rightarrow \infty$ (see Appendix A for the detailed derivation [133-136]). Here $\left|\cos k_{x}+m / t\right|<1$ is required for the localization (normalization) of these edge states. The obtained edge states are immune to disorder and hence topologically protected (see Appendix $B$ for details). Remarkably, the right edge state has the largest imaginary part for $\gamma>0$, whereas the left edge state has the smallest imaginary part. Physically, this results in the amplification (lasing) of the right edge state and the attenuation of the left edge state, just like a topological insulator laser recently proposed and realized [79.

In the gapless phases, there appear exceptional points on $k_{x}=0$ and/or $k_{x}= \pm \pi$ (Fig. 3(e-h)). Whereas no edge states appear in Fig. 3(e-f), a pair of chiral edge states emerges at both edges as shown in Fig. 3 (g-h), which are also described by Eqs. (17) and (18). The latter case corresponds to the gapless phase with $\nu_{0}, \nu_{\pi} \neq 0$ between the gapped $C=+1$ and $C=-1$ phases (see the green regions in Fig. 11). Such a gapless phase connects the gapped and topologically nontrivial phases, provided that $C_{4}$ rotational symmetry is maintained. Despite the absence of the energy gap, these chiral edge states are also stable against weak perturbations (see Appendix B for details), which implies their topological origin. We 
(a)

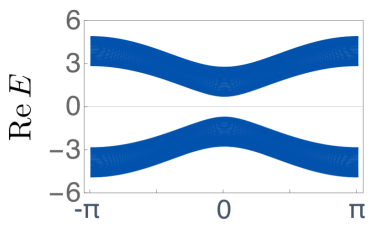

(c)

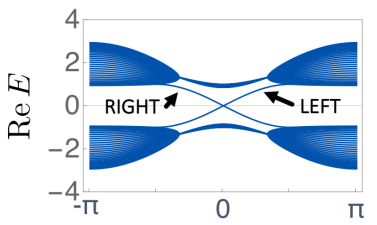

(e)

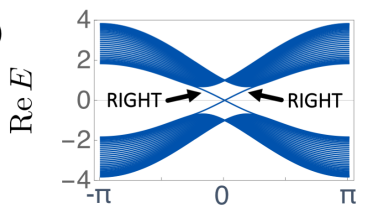

(g)

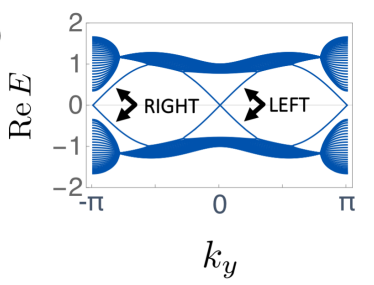

(b)

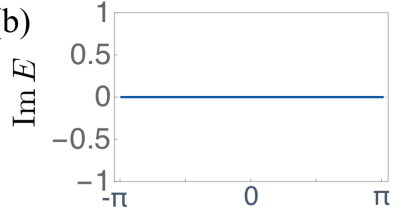

(d)

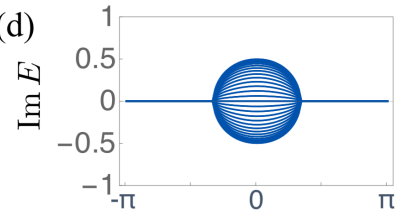

(f)

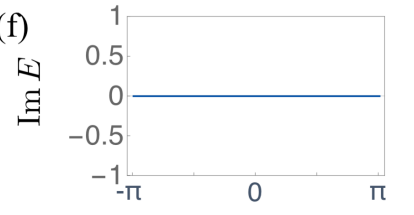

(h)

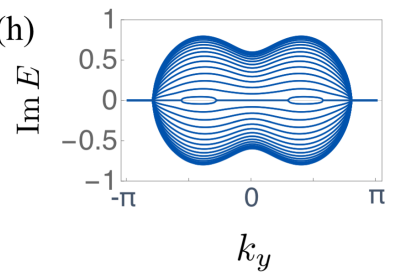

FIG. 5. Complex spectrum of the non-Hermitian Chern insulator given by Eq. 19, which has open boundaries in the $x$ direction $\left(L_{x}=30\right)$ and periodic boundaries in the $y$ direction. (a-b) Real and imaginary parts of the complex spectrum in the gapped and topologically trivial phase $(t=1.0, m=-3.0, \gamma=0.5)$. No gapless states appear between the gapped complex bands. (c-d) Real and imaginary parts of the complex spectrum in the gapped and topologically nontrivial phase $(t=1.0, m=-1.0, \gamma=0.5)$. A pair of chiral edge states appears between the gapped complex bands. (e-f) Real and imaginary parts of the complex spectrum for $t=1.0, m=-2.0$, and $\gamma=1.0$. Although the complex bands are gapless for the corresponding periodic systems (Fig. 2](e$\mathrm{f})$ ), the gap is open and the helical edge states localized only at the right edge appear. (g-h) Real and imaginary parts of the complex spectrum for $t=1.0, m=-0.2$, and $\gamma=1.0$. Although the complex bands are gapless for the corresponding periodic systems (Fig. 2(g-h)), the gap is open and two pairs of helical edge states appear, one localized at the right edge and the other localized at the left edge.

note that Ref. 137] discusses a similar gapless topological phase in Hermitian topological superconductors in two dimensions.

\section{B. Open boundaries in the $x$ direction}

We next consider the following non-Hermitian Chern insulator with open boundaries in the $x$ direction and

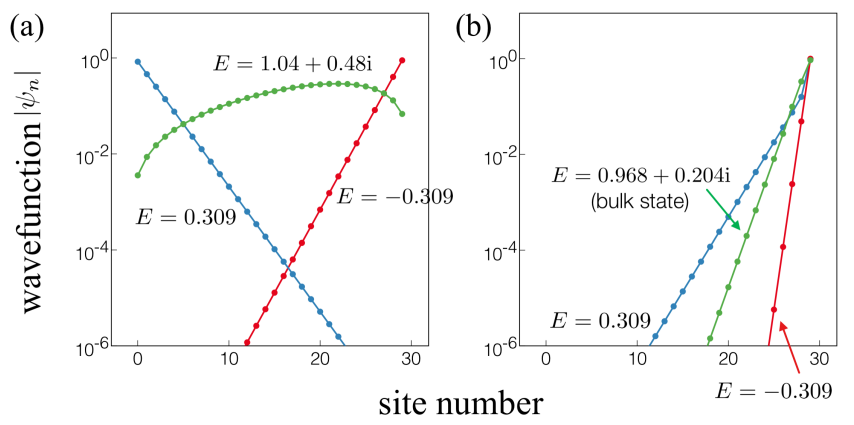

FIG. 6. Wavefunctions of the non-Hermitian Chern insulator given by Eq. (19), which has open boundaries in the $x$ direction $\left(L_{x}=30\right)$ and periodic boundaries in the $y$ direction. (a) The system is in the gapped and topologically nontrivial phase that corresponds to Fig. 5 (c-d) $(t=1.0, m=$ $\left.-1.0, \gamma=0.5 ; k_{x}=0.1 \pi\right)$. The chiral edge states with $E= \pm 0.309$ (red and blue dots) at both edges are more localized than the bulk state with $E=1.04+0.48$ i (green dots). (b) The system is in the gapped phase that corresponds to Fig. 5(e-f) $\left(t=1.0, m=-2.0, \gamma=1.0 ; k_{x}=0.1 \pi\right)$. The helical edge states with $E= \pm 0.309$ (red and blue dots) are localized only at the right edge, one of which is more localized than the bulk state with $E=0.968+0.204$ i (green dots) and the other of which is not.

periodic boundaries in the $y$ direction:

$$
\begin{aligned}
& \hat{H}=\sum_{x} \sum_{k_{y}}\left\{\left[\hat{c}_{x+1, k_{y}}^{\dagger} \frac{t\left(\sigma_{x}+\mathrm{i} \sigma_{y}\right)}{2} \hat{c}_{x, k_{y}}+\text { H.c. }\right]\right. \\
& \left.+\hat{c}_{x, k_{y}}^{\dagger}\left[\left(m+t \cos k_{y}\right) \sigma_{x}+\mathrm{i} \gamma \sigma_{y}+\left(t \sin k_{y}\right) \sigma_{z}\right] \hat{c}_{x, k_{y}}\right\},
\end{aligned}
$$

where $\hat{c}_{x, k_{y}}\left(\hat{c}_{x, k_{y}}^{\dagger}\right)$ annihilates (creates) a fermion with two internal degrees of freedom on site $x$ and with momentum $k_{y}$.

As in the case with the results obtained in the last section, the chiral edge states appear in the gapped phase with nonzero Chern number (Fig. 5. (c-d) and Fig. 6. (a)), whereas no edge states appear in the gapped phase with zero Chern number (Fig. $5(a-b))$. However, the energy dispersions are drastically different from those with periodic boundaries (Fig. 22 132, 138. In particular, the spectra are entirely real in some cases (Fig. 5(b, f)), which are to be contrasted with the corresponding periodic cases (Fig. 2(b, f)). The eigenstates also differ from the periodic case: all the eigenstates are localized at either left or right edge (Fig. 6). The energy dispersions of the chiral edge states are obtained as

$$
E_{\text {left }}\left(k_{y}\right)=t \sin k_{y}, \quad E_{\text {right }}\left(k_{y}\right)=-t \sin k_{y},
$$




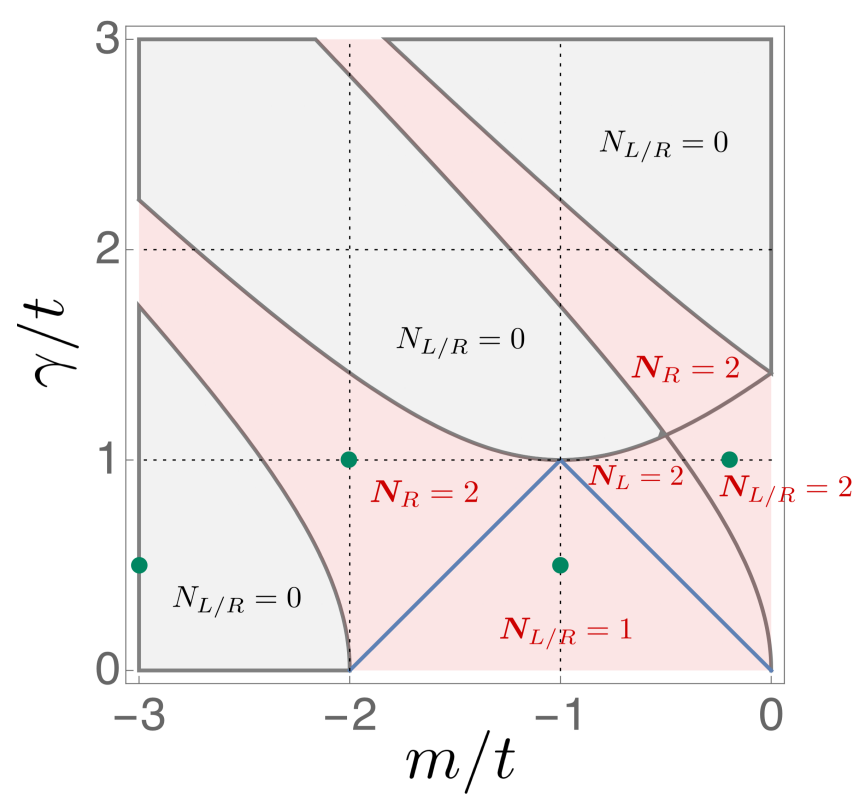

FIG. 7. Phase diagram of the non-Hermitian Chern insulator given by Eq. 19, which has open boundaries in the $x$ direction and periodic boundaries in the $y$ direction. The gapped phases are distinguished by the presence (red regions) or absence (gray regions) of the edge states, and $N_{L}\left(N_{R}\right)$ is the number of them localized at the left (right) edge. Across the blue lines, the number of edge states localized at either left or right edge changes, whereas the total number of edge states does not. The four green dots represent the set of parameters used in Fig. 5. In stark contrast to the Hermitian counterparts, the phase diagram is drastically different from that under the periodic boundary condition (Fig. 1).

and the corresponding edge states are given by

$$
\begin{gathered}
\hat{\Psi}_{\text {left }}\left(k_{y}\right) \propto \sum_{x=1}^{L_{x}}\left(-\cos k_{y}-\frac{m-\gamma}{t}\right)^{x-1} \hat{c}_{x, k_{y}}\left(\begin{array}{l}
1 \\
0
\end{array}\right), \\
\hat{\Psi}_{\text {right }}\left(k_{y}\right) \propto \sum_{x=1}^{L_{x}}\left(-\cos k_{y}-\frac{m+\gamma}{t}\right)^{x-1} \hat{c}_{x, k_{y}}\left(\begin{array}{l}
0 \\
1
\end{array}\right) .
\end{gathered}
$$

Here the imaginary parts of the edge states vanish in contrast to Eq. (17). In addition, the localization lengths depend on the non-Hermiticity $\gamma$ in contrast to Eq. 18], and they are different according to which edge they are localized at; the left edge state is present for $|m-\gamma|<2 t$, while the right edge state is present for $|m+\gamma|<2 t$.

Remarkably, the energy gap can be open (Fig. 5 (e-h)) even in the gapless phases for the corresponding periodic systems (Fig. 2(e-h)), and there appears a pair of helical edge states that are localized only at the right edge (Fig. 5(e-f) and Fig. 6(b)). The emergence of such anomalous edge states localized only at one edge reminds us of those in a one-dimensional system with sublattice symmetry 49 . In another phase, two pairs of helical edge states appear, one localized at the right edge and the other localized at the left edge (Fig. 5 (g-h)). These anomalous helical edge states are immune to disorder that does not close the energy gap, and hence they are topologically protected (see Appendix B for details). Moreover, the finite-size effects are negligible in this system (see Appendix $\mathrm{C}$ for details). The phase diagram of this open-boundary system is numerically obtained as shown in Fig. 7, which is drastically different from that of the periodic-boundary system (Fig. 1) in stark contrast to the Hermitian counterparts. Along with such sensitivity of the complex spectrum to the boundary conditions, recent work 67. proposed a generalized Chern number that precisely predicts the emergence of the chiral edge states even under the open boundary condition.

The emergence of the anomalous helical edge states is qualitatively understood as follows. Let us consider the topological phase transition from the gapped phase with $C=-1$ to that with $C=0$, through the $\nu_{0} \neq 0$ phase (Fig. 1). In the $C=-1$ phase, the chiral edge states appear at both edges given as Eq. (21), and all the eigenstates except for the left chiral edge state are localized at the right edge. If we approach the boundary between the $C=-1$ and $\nu_{0} \neq 0$ phases, the left chiral edge state gradually becomes delocalized, whereas the right chiral edge state does not. At the boundary between the $C=-1$ and $\nu_{0} \neq 0$ phases, the bulk gap closes (even under the open boundary condition along the $x$ direction), and the left chiral edge state is absorbed into the bulk eigenstates and shifted to the right edge. In fact, the left chiral edge state vanishes at the phase boundary $|m-\gamma|=2 t$, as described above. Inside the $\nu_{0} \neq 0$ phase, the bulk eigenstates acquire an energy gap, but the helical edge states remain gapless. At the boundary between the $\nu_{0} \neq 0$ and $C=0$ phases, the helical edge states are absorbed into the bulk eigenstates and disappear inside the $C=0$ phase.

Since the helical edge states emerge in the $\nu_{0} \neq 0$ phase, they may be related to the topological charges of exceptional points given by Eq. (7). In addition, we point out that the helical edge states in Fig. 5 (e-f) seem to bridge the two exceptional points that appear in the periodic systems (Fig. 2(e-f)).

\section{Open boundaries in both $x$ and $y$ directions}

Finally, we investigate the following non-Hermitian Chern insulator with open boundaries in both $x$ and $y$ directions:

$$
\begin{aligned}
\hat{H}=\sum_{x} \sum_{y}\{[ & \left.\hat{c}_{x, y+1}^{\dagger} \frac{t\left(\sigma_{x}+\mathrm{i} \sigma_{z}\right)}{2} \hat{c}_{x, y}+\text { H.c. }\right] \\
+ & {\left[\hat{c}_{x+1, y}^{\dagger} \frac{t\left(\sigma_{x}+\mathrm{i} \sigma_{y}\right)}{2} \hat{c}_{x, y}+\text { H.c. }\right] } \\
& \left.+\hat{c}_{x, y}^{\dagger}\left(m \sigma_{x}+\mathrm{i} \gamma \sigma_{y}\right) \hat{c}_{x, y}\right\},
\end{aligned}
$$

where $\hat{c}_{x, y}\left(\hat{c}_{x, y}^{\dagger}\right)$ annihilates (creates) a fermion with two internal degrees of freedom on site $(x, y)$. 


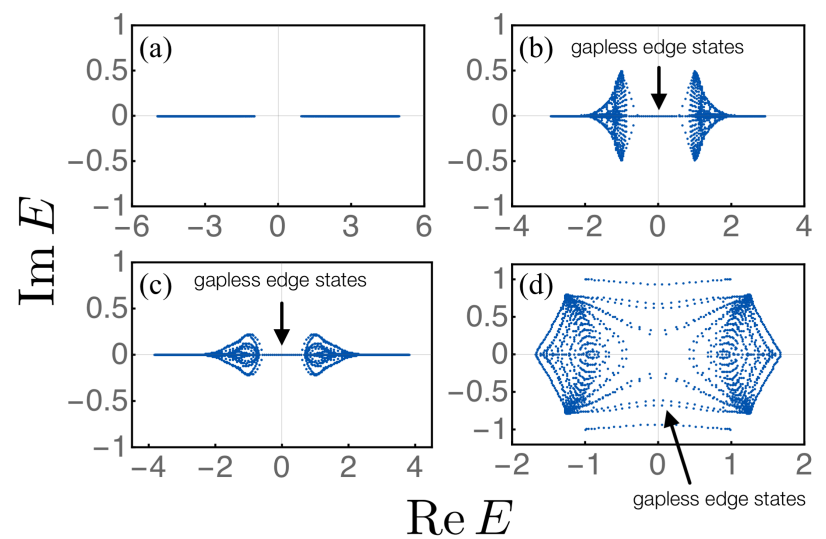

FIG. 8. Complex spectrum of the non-Hermitian Chern insulator given by Eq. (22), which has open boundaries in both $x$ and $y$ directions $\left(L_{x}=L_{y}=30\right)$. Real and imaginary parts of the complex eigenenergies are shown for (a) $t=1.0, m=$ $-3.0, \gamma=0.5$, (b) $t=1.0, m=-1.0, \gamma=0.5$, (c) $t=$ $1.0, m=-2.0, \gamma=1.0$, and (d) $t=1.0, m=-0.2, \gamma=1.0$. The complex gap is open in all the cases, and the gapless edge states appear for (b-d).
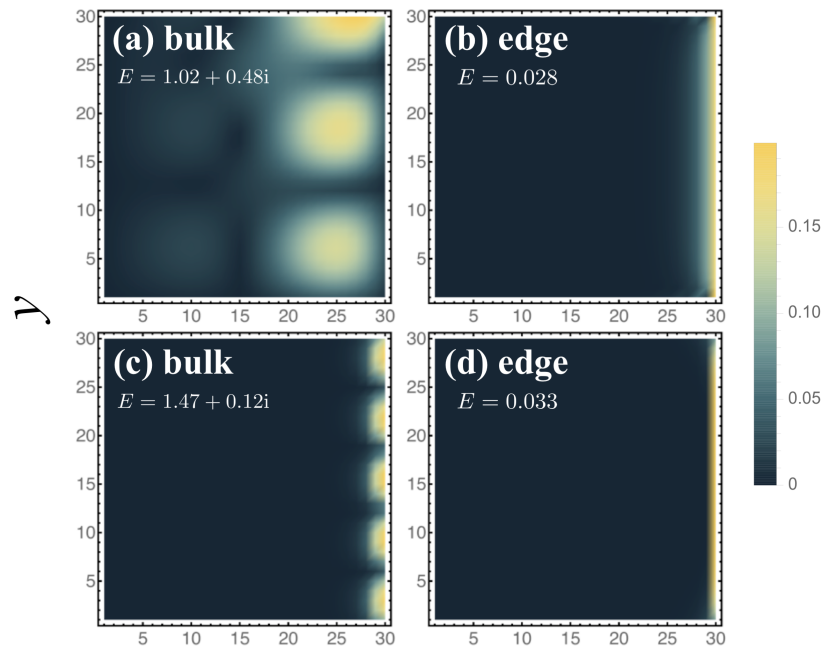

$x$

FIG. 9. Wavefunctions of the non-Hermitian Chern insulator given by Eq. 22, which has open boundaries in both $x$ and $y$ directions $\left(L_{x}=L_{y}=30\right)$. The system is in the gapped phase that corresponds to Fig. 8 (b) $(t=1.0, m=-1.0, \gamma=0.5)$ for (a-b) and Fig. 8 (c) $(t=1.0, m=-2.0, \gamma=1.0)$ for (c-d). The gapless states $(b, d)$ are more localized at the right edge than the bulk states $(\mathrm{a}, \mathrm{c})$.

The numerically obtained complex spectra are shown in Fig. 8. Remarkably, the gapless edge states do not hybridize with each other but survive even in this open boundary condition (Fig. 8(b-d)). These gapless states are more localized than the bulk states (Fig. 9). In contrast to the Hermitian counterparts, they are localized only at the right edge and hence do not circulate around the boundary.

\section{CONCLUSION AND OUTLOOK}

In this work, we have investigated a non-Hermitian Chern insulator and its bulk-boundary correspondence. We have found the phase diagram of the system with periodic boundaries; the gapped phases characterized by the Chern number are separated by the gapless phases characterized by the topological charges accompanied by exceptional points. We have also investigated topologically protected edge states for the same system with open boundaries. We have shown that the chiral edge states that correspond to the nontrivial topology of the bulk are robust even in this non-Hermitian system, and found the emergence of the anomalous helical edge states that are localized only at one edge. Our work serves as a two-dimensional generalization of the anomalous edge states in one dimension [49. Such anomalous edge states may appear even in different dimensions (including three dimensions) and symmetry classes, which we leave for future work.

Another important outstanding issue is to find the topological invariants that characterize the anomalous helical edge states and to establish their bulk-boundary correspondence. In the integer quantum Hall effect, the physical origin of the chiral edge states can be attributed to the presence of a confining potential around the edges and the gauge invariance for virtual magnetic fields [139142. It merits further study to establish such a physical picture for the helical edge states in the non-Hermitian Chern insulator discussed in this work.

Note added. - After completion of this work, we became aware of a recent related work [143], which also investigates a non-Hermitian Chern insulator and its edge states. Moreover, Refs. [144, 145], which appeared after the present work was submitted, study the Hall conductance of a non-Hermitian Chern insulator.

\section{ACKNOWLEDGMENT}

K. K. thanks Sho Higashikawa, Hosho Katsura, Tao Liu, Huan-Wen Wang, and Zhong Wang for helpful discussions. K. K. also appreciates hospitality of Condensed Matter Theory Group at Kyoto University through the domestic junior researcher exchange program of "Topological Materials Science," which inspired this work. This work was supported by KAKENHI Grant No. JP18H01145 and a Grant-in-Aid for Scientific Research on Innovative Areas "Topological Materials Science" (KAKENHI Grant No. JP15H05855) from the Japan Society for the Promotion of Science. K. K. was supported by the JSPS through Program for Leading Graduate Schools (ALPS). K. S. was supported by RIKEN Special Postdoctoral Researcher Program. 
(a)

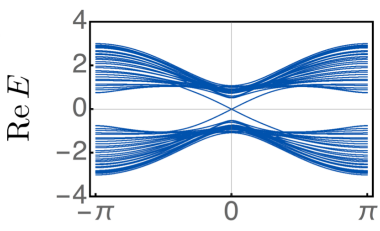

(c)

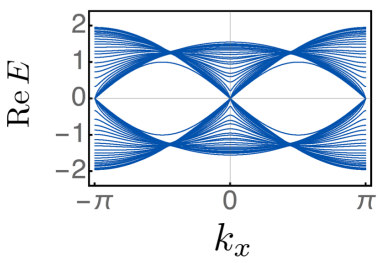

(e)

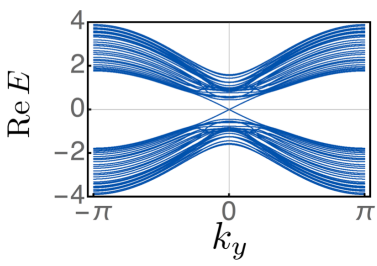

(b)

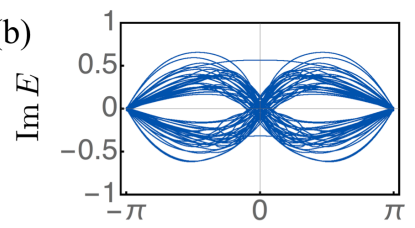

(d)

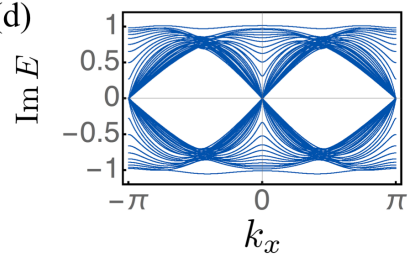

(f)

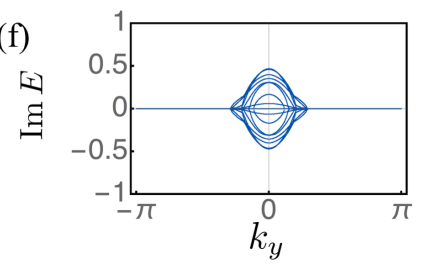

FIG. 10. Stability of the chiral and helical edge states. (ad) Complex spectrum of the disordered non-Hermitian Chern insulator given by Eq. (B1) as a function of the wavenumber $k_{x}$. The periodic boundary condition is imposed in the $x$ direction, while the open boundary condition is imposed in the $y$ direction $\left(L_{y}=30\right)$. The chiral edge states are robust against disorder for both (a-b) $t=1.0, m_{y}=-1.0+0.5 \epsilon_{y}$, and $\gamma_{y}=0.5+0.5 \epsilon_{y}^{\prime}$, and (c-d) $t=1.0, m_{y}=-0.2+0.2 \epsilon_{y}$, and $\gamma_{y}=1.0+0.2 \epsilon_{y}^{\prime}$, where $\epsilon_{y}$ and $\epsilon_{y}^{\prime}$ are random variables uniformly distributed over $[-0.5,0.5]$. (e-f) Complex spectrum of the disordered non-Hermitian Chern insulator given by Eq. B2 as a function of the wavenumber $k_{y}$. The open boundary condition is imposed in the $x$ direction $\left(L_{x}=30\right)$, while the periodic boundary condition is imposed in the $y$ direction. The helical edge states are robust against disorder for $t=1.0, m_{x}=-2.0+0.5 \epsilon_{x}$, and $\gamma_{x}=1.0+0.5 \epsilon_{x}^{\prime}$.

\section{Appendix A: Exact forms of the chiral edge states}

We analytically derive the energy dispersions and wave functions of the chiral edge states in the non-Hermitian Chern insulator discussed in the main text 133 . We first consider the chiral edge state at the left edge of the Hamiltonian given by Eq. (16). If the edge state is expressed as

$$
\hat{\Psi}_{\text {left }} \propto \sum_{y=1}^{L_{y}} \lambda^{y-1}\left(\hat{c}_{k_{x}, y} \vec{v}\right)
$$

where $\lambda$ denotes a parameter that determines the localization length (given by $-(\log |\lambda|)^{-1}$ ) and $\vec{v}$ is a two-component vector. Then the Schrödinger equation $\left[\hat{H}, \hat{\Psi}_{\text {left }}\right]=E_{\text {left }} \hat{\Psi}_{\text {left }}$ reduces to

$$
\left(\lambda^{-1} T+M+\lambda T^{\dagger}\right) \vec{v}=E_{\text {left }} \vec{v}
$$

in the bulk and

$$
\left(M+\lambda T^{\dagger}\right) \vec{v}=E_{\text {left }} \vec{v}
$$

(a)

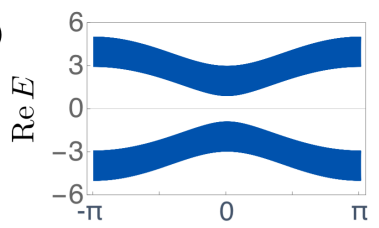

(c)

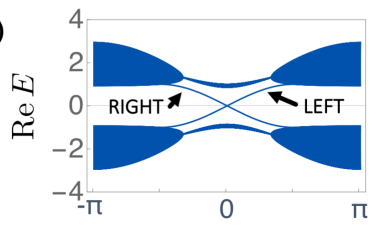

(e)

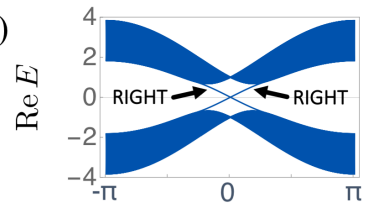

(g)

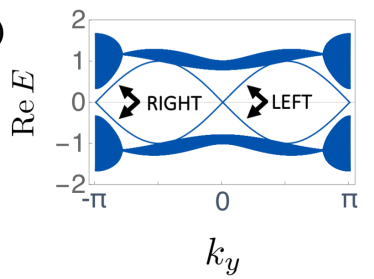

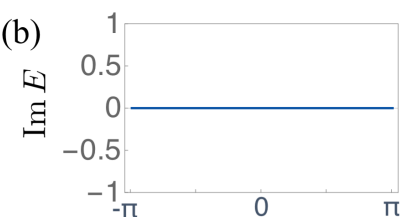

(d)

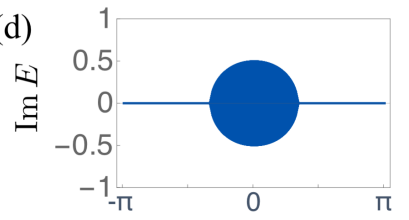

(f)

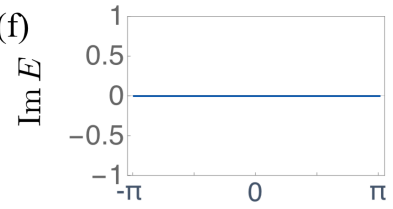

(h)

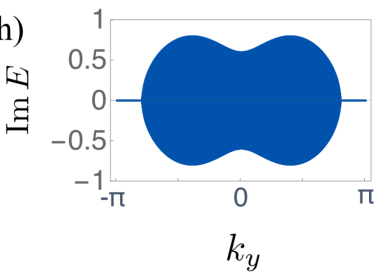

FIG. 11. Complex spectrum of the non-Hermitian Chern insulator given by Eq. (19), which has open boundaries in the $x$ direction $\left(L_{x}=200\right)$ and periodic boundaries in the $y$ direction. The same sets of parameters are used as in Fig. 5 The band gap is open and the chiral or helical edge states survive even in this larger system.

at the left edge. Here $T$ and $M$ are defined as $T:=t\left(\sigma_{x}+\mathrm{i} \sigma_{z}\right) / 2$ and $M:=\left(m+t \cos k_{x}\right) \sigma_{x}+$ $\left(\mathrm{i} \gamma+t \sin k_{x}\right) \sigma_{y}$, and we take the semi-infinite limit $L_{y} \rightarrow \infty$ and neglect the effect of the right edge. Combining Eqs. A2 and A3 , we have $T \vec{v}=0$, which leads to $\vec{v}=(1-\mathrm{i})^{T}$. Hence with Eq. A3 we have

$$
\begin{aligned}
{\left[E_{\text {left }}\right.} & \left.+\left(t \sin k_{x}+\mathrm{i} \gamma\right)\right](1-\mathrm{i})^{T} \\
& =\left[\lambda t+\left(m+t \cos k_{x}\right)\right](-\mathrm{i} 1)^{T},
\end{aligned}
$$

which gives Eqs. (17) and (18) in the main text. For the presence of this edge state, there exists a wavenumber $k_{x}$ that satisfies $\left|\cos k_{x}+m / t\right|<1$, which reduces to $|m|<2 t$. The other chiral edge state at the right edge is also obtained in a similar manner.

We next consider the chiral edge state at the left edge of the Hamiltonian given by Eq. (19). If the edge state is expressed as

$$
\hat{\Psi}_{\text {left }} \propto \sum_{x=1}^{L_{x}} \lambda_{\text {left }}^{x-1}\left(\hat{c}_{x, k_{y}} \vec{v}\right),
$$

the Schrödinger equation $\left[\hat{H}, \hat{\Psi}_{\text {left }}\right]=E_{\text {left }} \hat{\Psi}_{\text {left }}$ reduces to Eqs. A2 and A3 with $T:=t\left(\sigma_{x}+\mathrm{i} \sigma_{y}\right) / 2$ and 
$M:=\left(m+t \cos k_{y}\right) \sigma_{x}+\mathrm{i} \gamma \sigma_{y}+\left(t \sin k_{y}\right) \sigma_{z}$. We again have $T \vec{v}=0$ and hence $\vec{v}=\left(\begin{array}{ll}1 & 0\end{array}\right)^{T}$. With Eq. A3), we have

$$
\begin{aligned}
{\left[E_{\text {left }}\right.} & \left.-t \sin k_{y}\right]\left(\begin{array}{ll}
1 & 0
\end{array}\right)^{T} \\
& =\left[\lambda_{\text {left }} t+\left(m+t \cos k_{x}-\gamma\right)\right]\left(\begin{array}{lll}
0 & 1
\end{array}\right)^{T},
\end{aligned}
$$

which gives Eqs. 20) and (21) in the main text. While the other chiral edge state at the right edge is also obtained in a similar manner, their localizations depend differently on the non-Hermiticity $\gamma$; the left edge state appears for $|m-\gamma|<2 t$, while the right one appears for $|m+\gamma|<2 t$.

\section{Appendix B: Robustness against disorder}

The chiral and helical edge states revealed in the main text are immune to disorder. To confirm this, we first consider the following disordered non-Hermitian Chern insulator with periodic boundaries in the $x$ direction and open boundaries in the $y$ direction:

$$
\begin{aligned}
& \hat{H}=\sum_{k_{x}} \sum_{y}\left\{\left[\hat{c}_{k_{x}, y+1}^{\dagger} \frac{t\left(\sigma_{x}+\mathrm{i} \sigma_{z}\right)}{2} \hat{c}_{k_{x}, y}+\text { H.c. }\right]\right. \\
& \left.+\hat{c}_{k_{x}, y}^{\dagger}\left[\left(m_{y}+t \cos k_{x}\right) \sigma_{x}+\left(\mathrm{i} \gamma_{y}+t \sin k_{x}\right) \sigma_{y}\right] \hat{c}_{k_{x}, y}\right\},
\end{aligned}
$$

where $m_{y}$ and $\gamma_{y}$ denote the parameters characterizing disorder on site $y$. The chiral edge states appear even in the presence of disorder and hence are topologically protected (Fig. 10(a-d)). We also consider the following disordered non-Hermitian Chern insulator with open boundaries in the $x$ direction and periodic boundaries in the $y$ direction:

$$
\begin{aligned}
& \hat{H}=\sum_{x} \sum_{k_{y}}\left\{\left[\hat{c}_{x+1, k_{y}}^{\dagger} \frac{t\left(\sigma_{x}+\mathrm{i} \sigma_{y}\right)}{2} \hat{c}_{x, k_{y}}+\text { H.c. }\right]\right. \\
& \left.+\hat{c}_{x, k_{y}}^{\dagger}\left[\left(m_{x}+t \cos k_{y}\right) \sigma_{x}+\mathrm{i} \gamma_{x} \sigma_{y}+\left(t \sin k_{y}\right) \sigma_{z}\right] \hat{c}_{x, k_{y}}\right\},
\end{aligned}
$$

where $m_{x}$ and $\gamma_{x}$ denote the parameters characterizing disorder on site $x$. As well as the chiral edge states, the helical edge states are topologically protected (Fig. 10(ef)).

\section{Appendix C: Finite-size effect}

Figure 11 represents the complex spectrum of the nonHermitian Chern insulator with open boundaries in the $x$ direction and periodic boundaries in the $y$ direction. In contrast to Fig. 5 with $L_{x}=30$, Fig. 11 is on the larger systems with $L_{x}=200$. The band gap is open and the chiral or helical edge states survive even in this larger system; the finite-size effects are thus negligible.
[1] M. Z. Hasan and C. L. Kane, Colloquium: Topological insulators, Rev. Mod. Phys. 82, 3045 (2010).

[2] X.-L. Qi and S.-C. Zhang, Topological insulators and superconductors, Rev. Mod. Phys. 83, 1057 (2011).

[3] C.-K. Chiu, J. C. Y. Teo, A. P. Schnyder, and S. Ryu, Classification of topological quantum matter with symmetries, Rev. Mod. Phys. 88, 035005 (2016).

[4] B. A. Bernevig and T. L. Hughes, Topological Insulators and Topological Superconductors (Princeton University Press, Princeton, NJ, 2013).

[5] J. K. Asbóth, L. Oroszlány, and A. Pályi, A Short Course on Topological Insulators, Lecture Notes in Physics 919 (Springer, New York, 2016).

[6] D. J. Thouless, M. Kohmoto, M. P. Nightingale, and M. den Nijs, Quantized Hall conductance in a twodimensional periodic potential, Phys. Rev. Lett. 49, 405 (1982).

[7] F. D. M. Haldane, Model for a quantum Hall effect without Landau levels: Condensed-matter realization of the "parity anomaly", Phys. Rev. Lett. 61, 2015 (1988).

[8] C. L. Kane and E. J. Mele, $Z_{2}$ topological order and the quantum spin Hall effect, Phys. Rev. Lett. 95, 146802 (2005); Quantum spin Hall effect in graphene, Phys. Rev. Lett. 95, 226801 (2005).
[9] B. A. Bernevig, T. L. Hughes, and S.-C. Zhang, Quantum spin Hall effect and topological phase transition in HgTe quantum wells, Science 314, 1757 (2006).

[10] J. E. Moore and L. Balents, Topological invariants of time-reversal-invariant band structures, Phys. Rev. B 75, 121306(R) (2007).

[11] L. Fu and C. L. Kane, Topological insulators with inversion symmetry, Phys. Rev. B 76, 045302 (2007).

[12] M. König, S. Wiedmann, C. Brüne, A. Roth, H. Buhmann, L. Molenkamp, X.-L. Qi, and S.-C. Zhang, Quantum spin Hall insulator state in $\mathrm{HgTe}$ quantum wells, Science 318, 766 (2007).

[13] D. Hsieh, D. Qian, L. Wray, Y. Xia, Y. S. Hor, R. J. Cava, and M. Z. Hasan, A topological Dirac insulator in a quantum spin Hall phase, Nature 452, 970 (2008).

[14] N. Read and D. Green, Paired states of fermions in two dimensions with breaking of parity and timereversal symmetries and the fractional quantum Hall effect, Phys. Rev. B 61, 10267 (2000).

[15] A. Y. Kitaev, Unpaired Majorana fermions in quantum wires, Phys.-Usp. 44, 131 (2001).

[16] V. Mourik, K. Zuo, S. M. Frolov, S. R. Plissard, E. P. A. M. Bakkers, and L. P. Kouwenhoven, Signatures of Majorana fermions in hybrid superconductorsemiconductor nanowire devices, Science 336, 1003 
(2012).

[17] J. Alicea, New directions in the pursuit of Majorana fermions in solid state systems, Rep. Prog. Phys. 75, 076501 (2012).

[18] M. Sato and Y. Ando, Topological superconductors: a review, Rep. Prog. Phys. 80, 076501 (2017).

[19] X. Wan, A. M. Turner, A. Vishwanath, and S. Y. Savrasov, Topological semimetal and Fermi-arc surface states in the electronic structure of pyrochlore iridates, Phys. Rev. B 83, 205101 (2011).

[20] A. A. Burkov and L. Balents, Weyl semimetal in a topological insulator multilayer, Phys. Rev. Lett. 107, 127205 (2011).

[21] N. P. Armitage, E. J. Mele, and A. Vishwanath, Weyl and Dirac semimetals in three-dimensional solids, Rev. Mod. Phys. 90, 015001 (2018).

[22] F. D. M. Haldane and S. Raghu, Possible realization of directional optical waveguides in photonic crystals with broken time-reversal symmetry, Phys. Rev. Lett. 100, 013904 (2008).

[23] Z. Wang, Y. Chong, J. D. Joannopoulos, and M. Soljačić, Observation of unidirectional backscatteringimmune topological electromagnetic states, Nature 461, 772 (2009).

[24] M. Rechtsman, J. M. Zeuner, Y. Plotnik, Y. Lumer, D. Podolsky, F. Dreisow, S. Nolte, M. Segev, and A. Szameit, Photonic Floquet topological insulators, Nature 496, 196 (2013).

[25] L. Lu, J. D. Joannopoulos, and M. Soljačić, Topological photonics, Nat. Photon. 8, 821 (2014).

[26] T. Ozawa, H. M. Price, A. Amo, N. Goldman, M. Hafezi, L. Lu, M. Rechtsman, D. Schuster, J. Simon, O. Zilberberg, I. Carusotto, Topological photonics, arXiv: 1802.04173 .

[27] M. Atala, M. Aidelsburger, J. T. Barreiro, D. Abanin, T. Kitagawa, E. Demler, and I. Bloch, Direct measurement of the Zak phase in topological Bloch bands, Nat. Phys. 9, 795 (2013).

[28] G. Jotzu, M. Messer, R. Desbuquois, M. Lebrat, T. Uehlinger, D. Greif, and T. Esslinger, Experimental realization of the topological Haldane model with ultracold fermions, Nature 515, 237 (2014).

[29] M. Aidelsburger, M. Lohse, C. Schweizer, M. Atala, J. T. Barreiro, S. Nascimbène, N. R. Cooper, I. Bloch, and N. Goldman, Measuring the Chern number of Hofstadter bands with ultracold bosonic atoms, Nat. Phys. 11, 162 (2015).

[30] S. Nakajima, T. Tomita, S. Taie, T. Ichinose, H. Ozawa, L. Wang, M. Troyer, and Y. Takahashi, Topological Thouless pumping of ultracold fermions, Nat. Phys. 12, 296 (2016).

[31] M. Lohse, C. Schweizer, O. Zilberberg, M. Aidelsburger, and I. Bloch, A Thouless quantum pump with ultracold bosonic atoms in an optical superlattice, Nat. Phys. 12, 350 (2016).

[32] N. Goldman, J. C. Budich, and P. Zoller, Topological quantum matter with ultracold gases in optical lattices, Nat. Phys. 12, 639 (2016).

[33] N. R. Cooper, J. Dalibard, and I. B. Spielman, Topological bands for ultracold atoms, arXiv: 1803.00249.

[34] X.-L. Qi, T. L. Hughes, and S.-C. Zhang, Topological field theory of time-reversal invariant insulators, Phys. Rev. B 78, 195424 (2008).
[35] A. P. Schnyder, S. Ryu, A. Furusaki, and A. W. W Ludwig, Classification of topological insulators and superconductors in three spatial dimensions, Phys. Rev. B 78, 195125 (2008).

[36] A. Kitaev, Periodic table for topological insulators and superconductors, AIP Conf. Proc. 1134, 22 (2009).

[37] S. Ryu, A. P. Schnyder, A. Furusaki, and A. W. Ludwig, Topological insulators and superconductors: tenfold way and dimensional hierarchy, New J. Phys. 12, 065010 (2010).

[38] J. C. Y. Teo and C. L. Kane, Topological defects and gapless modes in insulators and superconductors, Phys. Rev. B 82, 115120 (2010).

[39] R.-J. Slager, A. Mesaros, V. Juriŭić, and J. Zaanen, The space group classification of topological band-insulators, Nat. Phys. 9, 98 (2013).

[40] K. Shiozaki and M. Sato, Topology of crystalline insulators and superconductors, Phys. Rev. B 90, 165114 (2014).

[41] J. Kruthoff, J. de Boer, J. van Wezel, C. K. Kane, and R.-J. Slager, Topological classification of crystalline insulators through band structure combinatorics, Phys. Rev. X 7, 041069 (2017).

[42] M. S. Rudner and L. S. Levitov, Topological transition in a non-Hermitian quantum walk, Phys. Rev. Lett. 102, 065703 (2009).

[43] Y. C. Hu and T. L. Hughes, Absence of topological insulator phases in non-Hermitian PT-symmetric Hamiltonians, Phys. Rev. B 84, 153101 (2011).

[44] K. Esaki, M. Sato, K. Hasebe, and M. Kohmoto, Edge states and topological phases in non-Hermitian systems, Phys. Rev. B 84, 205128 (2011).

[45] S.-D. Liang and G.-Y. Huang, Topological invariance and global Berry phase in non-Hermitian systems, Phys. Rev. A 87, 012118 (2013).

[46] H. Schomerus, Topologically protected midgap states in complex photonic lattices, Opt. Lett. 38, 1912 (2013).

[47] S. Malzard, C. Poli, and H. Schomerus, Topologically protected defect states in open photonic systems with non-Hermitian charge-conjugation and parity-time symmetry, Phys. Rev. Lett. 115, 200402 (2015).

[48] P. San-Jose, J. Cayao, E. Prada, and R. Aguado, $M a-$ jorana bound states from exceptional points in nontopological superconductors, Sci. Rep. 6, 21427 (2016).

[49] T. E. Lee, Anomalous edge state in a non-Hermitian lattice, Phys. Rev. Lett. 116, 133903 (2016).

[50] A. K. Harter, T. E. Lee, and Y. N. Joglekar, PT breaking threshold in spatially asymmetric Aubry-André and Harper models: Hidden symmetry and topological states, Phys. Rev. A 93, 062101 (2016).

[51] D. Leykam, K. Y. Bliokh, C. Huang, Y. D. Chong, and F. Nori, Edge modes, degeneracies, and topological numbers in non-Hermitian systems, Phys. Rev. Lett. 118, 040401 (2017).

[52] Y. Xu, S.-T. Wang, and L.-M. Duan, Weyl exceptional rings in a three-dimensional dissipative cold atomic gas, Phys. Rev. Lett. 118, 045701 (2017).

[53] H. Menke and M. Hirschmann, Topological quantum wires with balanced gain and loss, Phys. Rev. B 95, 174506 (2017).

[54] J. González and R. A. Molina, Topological protection from exceptional points in Weyl and nodal-line semimetals, Phys. Rev. B 96, 045437 (2017). 
[55] L. Campos Venuti, Z. Ma, H. Saleur, and S. Haas, Topological protection of coherence in a dissipative environment, Phys. Rev. A 96, 053858 (2017).

[56] S. Lieu, Topological phases in the non-Hermitian SuSchrieffer-Heeger model, Phys. Rev. B 97, 045106 (2018).

[57] A. A. Zyuzin and Z. Yu. Zyuzin, Flat band in disorderdriven non-Hermitian Weyl semimetals, Phys. Rev. B 97, 041203(R) (2018).

[58] A. Cerjan, M. Xiao, L. Yuan, and S. Fan, Effects of nonHermitian perturbations on Weyl Hamiltonians with arbitrary topological charges, Phys. Rev. B 97, 075128 (2018).

[59] V. M. Martinez Alvarez, J. E. Barrios Vargas, and L. E. F. Foa Torres, Non-Hermitian robust edge states in one dimension: Anomalous localization and eigenspace condensation at exceptional points, Phys. Rev. B 97, 121401(R) (2018).

[60] Y. Xiong, Why does bulk boundary correspondence fail in some non-hermitian topological models, J. Phys. Commun. 2, 035043 (2018).

[61] R. A. Molina and J. González, Surface and 3D quantum Hall effects from engineering of exceptional points in nodal-line semimetals, Phys. Rev. Lett. 120, 146601 (2018).

[62] H. Shen, B. Zhen, and L. Fu, Topological band theory for non-Hermitian Hamiltonians, Phys. Rev. Lett. 120, 146402 (2018).

[63] C. Yin, H. Jiang, L. Li, R. Lü, and S. Chen, Geometrical meaning of winding number and its characterization of topological phases in one-dimensional chiral nonHermitian systems, Phys. Rev. A 97, 052115 (2018).

[64] H. Shen and L. Fu, Quantum oscillation from in-gap states and a non-Hermitian Landau level problem, Phys. Rev. Lett. 121, 026403 (2018).

[65] T. Yoshida, R. Peters, and N. Kawakami, NonHermitian perspective of the band structure in heavyfermion systems, Phys. Rev. B 98, 035141 (2018).

[66] K. Kawabata, Y. Ashida, H. Katsura, and M. Ueda, Parity-time-symmetric topological superconductor, Phys. Rev. B 98, 085116 (2018).

[67] S. Yao and Z. Wang, Edge states and topological invariants of non-Hermitian systems, Phys. Rev. Lett. 121, 086803 (2018); S. Yao, F. Song, and Z. Wang, NonHermitian Chern bands, Phys. Rev. Lett. 121, 136802 (2018).

[68] Z. Gong, Y. Ashida, K. Kawabata, K. Takasan, S. Higashikawa, and M. Ueda, Topological phases of nonHermitian systems, Phys. Rev. X 8, 031079 (2018).

[69] X. Ni, D. Smirnova, A. Poddubny, D. Leykam, Y. Chong, and A. B. Khanikaev, $\mathcal{P} \mathcal{T}$ phase transitions of edge states at $\mathcal{P} \mathcal{T}$ symmetric interfaces in nonHermitian topological insulators, Phys. Rev. B 98, 165129 (2018).

[70] V. Kozii and L. Fu, Non-Hermitian topological theory of finite-lifetime quasiparticles: Prediction of bulk Fermi arc due to exceptional point, arXiv: 1708.05841.

[71] M. Papaj, H. Isobe, and L. Fu, Nodal arc in disordered Dirac fermions: Connection to non-Hermitian band theory, arXiv: 1802.00443.

[72] K. Kawabata, S. Higashikawa, Z. Gong, Y. Ashida, and M. Ueda, Topological unification of time-reversal and particle-hole symmetries in non-Hermitian physics, arXiv: 1804.04676 .
[73] J. M. Zeuner, M. C. Rechtsman, Y. Plotnik, Y. Lumer, S. Nolte, M. S. Rudner, M. Segev, and A. Szameit, Observation of a topological transition in the bulk of a nonHermitian system, Phys. Rev. Lett. 115, 040402 (2015).

[74] S. Weimann, M. Kremer, Y. Plotnik, Y. Lumer, S. Nolte, K. G. Makris, M. Segev, M. C. Rechtsman, and A. Szameit, Topologically protected bound states in photonic parity-time-symmetric crystals, Nat. Mater. 16, 433 (2017).

[75] D. Kim, K. Mochizuki, N. Kawakami, and H. Obuse, Floquet topological phases driven by $\mathcal{P} \mathcal{T}$ symmetric nonunitary time evolution, arXiv: 1609.09650; L. Xiao, X. Zhan, Z. H. Bian, K. K. Wang, X. Zhang, X. P. Wang, J. Li, K. Mochizuki, D. Kim, N. Kawakami, W. Yi, H. Obuse, B. C. Sanders, and P. Xue, Observation of topological edge states in parity-time-symmetric quantum walks, Nat. Phys. 13, 1117 (2017).

[76] H. Zhou, C. Peng, Y. Yoon, C. W. Hsu, K. A. Nelson, L. Fu, J. D. Joannopoulos, M. Soljačić, and B. Zhen, Observation of bulk Fermi arc and polarization half charge from paired exceptional points, Science 359, 1009 (2018).

[77] H. Zhao, P. Miao, M. H. Teimourpour, S. Malzard, R. El-Ganainy, H. Schomerus, and L. Feng, Topological hybrid silicon microlasers, Nat. Commun. 9, 981 (2018).

[78] M. Parto, S. Wittek, H. Hodaei, G. Harari, M. A. Bandres, J. Ren, M. C. Rechtsman, M. Segev, D. N. Christodoulides, and M. Khajavikhan, Edge-mode lasing in $1 D$ topological active arrays, Phys. Rev. Lett. 120, 113901 (2018).

[79] G. Harari, M. A. Bandres, Y. Lumer, M. C. Rechtsman, Y. D. Chong, M. Khajavikhan, D. N. Christodoulides, and M. Segev, Topological insulator laser: Theory, Science 359, eaar4003 (2018); M. A. Bandres, S. Wittek, G. Harari, M. Parto, J. Ren, M. Segev, D. Christodoulides, and M. Khajavikhan, Topological insulator laser: Experiments, Science 359, eaar4005 (2018).

[80] M. Pan, H. Zhao, P. Miao, S. Longhi, and L. Feng, Photonic zero mode in a non-Hermitian photonic lattice, Nat. Commun. 9, 1308 (2018).

[81] C. M. Bender, Making sense of non-Hermitian Hamiltonians, Rep. Prog. Phys. 70, 947 (2007).

[82] V. V. Konotop, J. Yang, and D. A. Zezyulin, Nonlinear waves in $\mathcal{P} \mathcal{T}$-symmetric systems, Rev. Mod. Phys. 88, 035002 (2016).

[83] L. Feng, R. El-Ganainy, and L. Ge, Non-Hermitian photonics based on parity-time symmetry, Nat. Photon. 11, 752 (2017).

[84] R. El-Ganainy, K. G. Makris, M. Khajavikhan, Z. H. Musslimani, S. Rotter, and D. N. Christodoulides, NonHermitian physics and PT symmetry, Nat. Phys. 14, 11 (2018).

[85] N. Hatano and D. R. Nelson, Localization transitions in non-Hermitian quantum mechanics, Phys. Rev. Lett. 77, 570 (1996).

[86] C. M. Bender and S. Boettcher, Real spectra in nonHermitian Hamiltonians having $\mathcal{P} \mathcal{T}$ symmetry, Phys. Rev. Lett. 80, 5243 (1998).

[87] C. M. Bender, D. C. Brody, H. F. Jones, and B. K. Meister, Faster than Hermitian quantum mechanics, Phys. Rev. Lett. 98, 040403 (2007).

[88] Z. H. Musslimani, K. G. Makris, R. El-Ganainy, and D. N. Christodoulides, Optical solitons in PT periodic potentials, Phys. Rev. Lett. 100, 030402 (2008). 
[89] K. G. Makris, R. El-Ganainy, D. N. Christodoulides, and Z. H. Musslimani, Beam dynamics in $\mathcal{P} \mathcal{T}$ symmetric optical lattices, Phys. Rev. Lett. 100, 103904 (2008).

[90] E.-M. Graefe, H. J. Korsch, and A. E. Niederle, Meanfield dynamics of a non-Hermitian Bose-Hubbard dimer, Phys. Rev. Lett. 101, 150408 (2008).

[91] U. Günther and B. F. Samsonov, Naimark-dilated PT-symmetric brachistochrone, Phys. Rev. Lett. 101, 230404 (2008).

[92] S. Longhi, Bloch oscillations in complex crystals with $\mathcal{P} \mathcal{T}$ symmetry, Phys. Rev. Lett. 103, 123601 (2009).

[93] S. Longhi, $\mathcal{P} \mathcal{T}$-symmetric laser absorber, Phys. Rev. A 82, 031801(R) (2010).

[94] Y. D. Chong, L. Ge, and A. D. Stone, $\mathcal{P} \mathcal{T}$-symmetry breaking and laser-absorber modes in optical scattering systems, Phys. Rev. Lett. 106, 093902 (2011).

[95] Z. Lin, H. Ramezani, T. Eichelkraut, T. Kottos, H. Cao, and D. N. Christodoulides, Unidirectional invisibility induced by $\mathcal{P} \mathcal{T}$-symmetric periodic structures, Phys. Rev. Lett. 106, 213901 (2011).

[96] A. Szameit, M. C. Rechtsman, O. Bahat-Treidel, and M. Segev, $\mathcal{P} \mathcal{T}$-symmetry in honeycomb photonic lattices, Phys. Rev. A 84, 021806(R) (2011).

[97] D. C. Brody and E.-M. Graefe, Mixed-state evolution in the presence of gain and loss, Phys. Rev. Lett. 109, 230405 (2012).

[98] J. Wiersig, Enhancing the sensitivity of frequency and energy splitting detection by using exceptional points: Application to microcavity sensors for single-particle detection, Phys. Rev. Lett. 112, 203901 (2014).

[99] T. E. Lee and C.-K. Chan, Heralded magnetism in nonHermitian atomic systems, Phys. Rev. X 4, 041001 (2014).

[100] T. E. Lee, F. Reiter, and N. Moiseyev, Entanglement and spin squeezing in non-Hermitian phase transitions, Phys. Rev. Lett. 113, 250401 (2014).

[101] V. Tripathi, A. Galda, H. Barman, and V. M. Vinokur, Parity-time symmetry-breaking mechanism of dynamic Mott transitions in dissipative systems, Phys. Rev. B 94, 041104(R) (2016).

[102] Z.-P. Liu, J. Zhang, S. K. Özdemir, B. Peng, H. Jing, X.-Y. Lü, C.-W. Li, L. Yang, F. Nori, and Y.-x. Liu, Metrology with $\mathcal{P} \mathcal{T}$-symmetric cavities: Enhanced sensitivity near the $\mathcal{P} \mathcal{T}$-phase transition, Phys. Rev. Lett. 117, 110802 (2016).

[103] Y. Ashida, S. Furukawa, and M. Ueda, Quantum critical behavior influenced by measurement backaction in ultracold gases, Phys. Rev. A 94, 053615 (2016); Parity-timesymmetric quantum critical phenomena, Nat. Commun. 8, 15791 (2017).

[104] L. Ge, Symmetry-protected zero-mode laser with a tunable spatial profile, Phys. Rev. A 95, 023812 (2017).

[105] S. Yin, G.-Y. Huang, C.-Y. Lo, and P. Chen, KibbleZurek scaling in the Yang-Lee edge singularity, Phys. Rev. Lett. 118, 065701 (2017).

[106] K. Kawabata, Y. Ashida, and M. Ueda, Information retrieval and criticality in parity-time-symmetric systems, Phys. Rev. Lett. 119, 190401 (2017).

[107] B. Qi, L. Zhang, and L. Ge, Defect states emerging from a non-Hermitian flatband of photonic zero modes, Phys. Rev. Lett. 120, 093901 (2018).

[108] V. V. Konotop and D. A. Zezyulin, Odd-time reversal $\mathcal{P} \mathcal{T}$ symmetry induced by an anti- $\mathcal{P} \mathcal{T}$-symmetric medium, Phys. Rev. Lett. 120, 123902 (2018).

[109] Y. Ashida and M. Ueda, Full-counting many-particle $d y$ namics: Nonlocal and chiral propagation of correlations, Phys. Rev. Lett. 120, 185301 (2018).

[110] F. Quijandría, U. Naether, S. K. Özdemir, F. Nori, and D. Zueco, $\mathcal{P} \mathcal{T}$-symmetric circuit- $Q E D$, Phys. Rev. A 97, 053846 (2018).

[111] D. Trypogeorgos, A. Valdés-Curiel, I. B. Spielman, and C. Emary, Perpetual emulation threshold of $\mathcal{P} \mathcal{T}$ symmetric Hamiltonians, J. Phys. A 51, 325302 (2018).

[112] J. A. S. Lourenço, R. L. Eneias, and R. G. Pereira, Kondo effect in a $\mathcal{P} \mathcal{T}$-symmetric non-Hermitian Hamiltonian, Phys. Rev. B 98, 085126 (2018).

[113] M. Nakagawa, N. Kawakami, and M. Ueda, NonHermitian Kondo effect in ultracold alkaline-earth atoms, arXiv: 1806.04039.

[114] A. Guo, G. J. Salamo, D. Duchesne, R. Morandotti, M. Volatier-Ravat, V. Aimez, G. A. Siviloglou, and D. N. Christodoulides, Observation of $\mathcal{P} \mathcal{T}$-symmetry breaking in complex optical potentials, Phys. Rev. Lett. 103, 093902 (2009).

[115] C. E. Rüter, K. G. Makris, R. El-Ganainy, D. N. Christodoulides, M. Segev, and D. Kip, Observation of parity-time symmetry in optics, Nat. Phys. 6, 192 (2010).

[116] A. Regensburger, C. Bersch, M.-A. Miri, G. Onishchukov, D. N. Christodoulides, and U. Peschel, Parity-time synthetic photonic lattices, Nature 488, 167 (2012).

[117] L. Feng, Y.-L. Xu, W. S. Fegadolli, M.-H. Lu, J. E. B. Oliveira, V. R. Almeida, Y.-F. Chen, and A. Scherer, Experimental demonstration of a unidirectional reflectionless parity-time metamaterial at optical frequencies, Nat. Mater. 12, 108 (2013).

[118] B. Peng, Ş. K. Özdemir, F. Lei, F. Monifi, M. Gianfreda, G. L. Long, S. Fan, F. Nori, C. M. Bender, and L. Yang, Parity-time-symmetric whispering-gallery microcavities, Nat. Phys. 10, 394 (2014).

[119] L. Feng, Z. J. Wong, R.-M. Ma, Y. Wang, and X. Zhang, Single-mode laser by parity-time symmetry breaking, Science 346, 972 (2014).

[120] H. Hodaei, M.-A. Miri, M. Heinrich, D. N. Christodoulides, and M. Khajavikhan, Parity-timesymmetric microring lasers, Science 346, 975 (2014).

[121] B. Zhen, C. W. Hsu, Y. Igarashi, L. Lu, I. Kaminer, A. Pick, S.-L. Chua, J. D. Joannopoulos, and M. Soljačić, Spawning rings of exceptional points out of Dirac cones, Nature 525, 354 (2015).

[122] T. Gao, E. Estrecho, K. Y. Bliokh, T. C. H. Liew, M. D. Fraser, S. Brodbeck, M. Kemp, C. Schneider, S. Höfling, Y. Yamamoto, F. Nori, Y. S. Kivshar, A. G. Truscott, R. G. Dall, and E. A. Ostrovskaya, Observation of nonHermitian degeneracies in a chaotic exciton-polariton billiard, Nature 526, 554 (2015).

[123] J.-S. Tang, Y. T. Wang, S. Yu, D. Y. He, J. S. Xu, B. H. Liu, G. Chen, Y. N. Sun, K. Sun, Y. J. Han, C.-F. $\mathrm{Li}$, and G.-G. Guo, Experimental investigation of the no-signaling principle in parity-time symmetric theory using an open quantum system, Nat. Photon. 10, 642 (2016).

[124] P. Peng, W. Cao, C. Shen, W. Qu, J. Wen, L. Jiang, and Y. Xiao, Anti-parity-time symmetry with flying atoms, Nat. Phys. 12, 1139 (2016). 
[125] H. Hodaei, A. U. Hassan, S. Wittek, H. Garcia-Gracia, R. El-Ganainy, D. N. Christodoulides, and M. Khajavikhan, Enhanced sensitivity at higher-order exceptional points, Nature 548, 187 (2017).

[126] W. Chen, Ş. K. Özdemir, G. Zhao, J. Wiersig, and L. Yang, Exceptional points enhance sensing in an optical microcavity, Nature 548, 192 (2017).

[127] M. V. Berry, Physics of nonhermitian degeneracies, Czech. J. Phys. 54, 1039 (2004).

[128] W. D. Heiss, The physics of exceptional points, J. Phys. A 45, 444016 (2012).

[129] N. Moiseyev, Non-Hermitian quantum mechanics (Cambridge University Press, Cambridge, England, 2011).

[130] D. C. Brody, Biorthogonal quantum mechanics, J. Phys. A 47, 035305 (2014).

[131] D. C. Brody, Consistency of PT-symmetric quantum mechanics, J. Phys. A 49, 10LT03 (2016).

[132] Such behavior unique to non-Hermitian systems was also reported in Refs. 49, 59, 60, 67, 68.

[133] A systematic way to analytically obtain the edge states is described in Refs. [134-136.

[134] R. S. K. Mong and V. Shivamoggi, Edge states and the bulk-boundary correspondence in Dirac Hamiltonians, Phys. Rev. B 83, 125109 (2011).

[135] A. Alase, E. Cobanera, G. Ortiz, and L. Viola, Exact solution of quadratic fermionic Hamiltonians for arbitrary boundary conditions, Phys. Rev. Lett. 117, 076804 (2016); Generalization of Bloch's theorem for arbitrary boundary conditions: Theory, Phys. Rev. B 96, 195133 (2017).

[136] K. Kawabata, R. Kobayashi, N. Wu, and H. Katsura, Exact zero modes in twisted Kitaev chains, Phys. Rev. B 95, 195140 (2017).
[137] M. Sato and S. Fujimoto, Existence of Majorana fermions and topological order in nodal superconductors with spin-orbit interactions in external magnetic fields, Phys. Rev. Lett. 105, 217001 (2010).

[138] We observe that such qualitative change of the complex spectrum does not occur for the system with open boundaries only in the $y$ direction. In addition, all the eigenstates except the chiral edge states are delocalized in the bulk in this case.

[139] R. B. Laughlin, Quantized Hall conductivity in two dimensions, Phys. Rev. B 23, 5632(R) (1981).

[140] B. I. Halperin, Quantized Hall conductance, currentcarrying edge states, and the existence of extended states in a two-dimensional disordered potential, Phys. Rev. B 25, 2185 (1982).

[141] M. Büttiker, Absence of backscattering in the quantum Hall effect in multiprobe conductors, Phys. Rev. B 38, 9375 (1988).

[142] Y. Hatsugai, Edge states in the integer quantum Hall effect and the Riemann surface of the Bloch function, Phys. Rev. B 48, 11851 (1993); Chern number and edge states in the integer quantum Hall effect, Phys. Rev. Lett. 71, 3697 (1993);

[143] F. K. Kunst, E. Edvardsson, J. C. Budich, and E. J. Bergholtz, Biorthogonal bulk-boundary correspondence in non-Hermitian systems, Phys. Rev. Lett. 121, 026808 (2018).

[144] T. M. Philip, M. R. Hirsbrunner, and M. J. Gilbert, Loss of Hall conductivity quantization in a non-Hermitian quantum anomalous Hall insulator, Phys. Rev. B 98, 155430 (2018).

[145] Y. Chen and H. Zhai, Hall conductance of a nonHermitian Chern insulator, arXiv: 1806.06566. 